\title{
Geographic access to radiation therapy facilities and disparities of early-stage breast cancer treatment
}

\author{
Yan Lin, ${ }^{1}$ Michael C. Wimberly, ${ }^{2}$ Patricia Da Rosa, ${ }^{3}$ Joseph Hoover, ${ }^{4}$ William F. Athas ${ }^{5}$ \\ ${ }^{1}$ Department of Geography and Environmental Studies, University of New Mexico, Albuquerque, NM; \\ ${ }^{2}$ Geospatial Sciences Center of Excellence, South Dakota State University, Brookings, SD; ${ }^{3}$ Office of \\ Research, College of Nursing, South Dakota State University, Brookings, SD; ${ }^{4}$ Community Environmental \\ Health Program, College of Pharmacy, University of New Mexico, Albuquerque, NM; ${ }^{5}$ College of \\ Population Health, University of New Mexico, Albuquerque, NM, USA
}

\begin{abstract}
Few studies of breast cancer treatment have focused on the Northern Plains of the United States, an area with a high mastectomy rate. This study examined the association between geographic access to radiation therapy facilities and receipt of breast cancer treatments among early-stage breast cancer patients in South
\end{abstract}

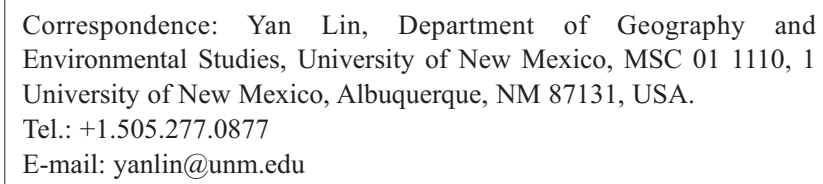

Key words: Geographic access; Radiation therapy; Breast cancer; Breast conserving surgery; Mastectomy; South Dakota.

Acknowledgements: the authors thank the South Dakota Department of Health and the South Dakota Cancer Registry for providing the data used in the research. We are grateful to Ms. Kay Dosch and anonymous reviewers from the South Dakota Cancer Registry for providing invaluable suggestions on this manuscript.

Contributions: all authors contributed to the study design, writing and revision of the article. YL designed and carried out the analysis. All authors read and approved the final manuscript.

Conflict of interest: the authors declare no potential conflict of interest.

Funding: none.

Ethical approval: the use and analysis of this breast cancer database have been approved by the institutional review board of the South Dakota Cancer Registry and South Dakota State University.

Received for publication: 5 September 2017.

Revision received: 13 November 2017.

Accepted for publication: 17 November 2017.

CCopyright Y. Lin et al., 2018

Licensee PAGEPress, Italy

Geospatial Health 2018; 13:622

doi:10.4081/gh.2018.622

This article is distributed under the terms of the Creative Commons Attribution Noncommercial License (CC BY-NC 4.0) which permits any noncommercial use, distribution, and reproduction in any medium, provided the original author(s) and source are credited.
Dakota. Based on 4,209 early-stage breast cancer patients diagnosed between 2001 and 2012 in South Dakota, the study measured geographic proximity to radiation therapy facilities using the shortest travel time for patients to the closest radiation therapy facility. Two-level logistic regression models were used to estimate for early stage cases i) the odds of mastectomy versus breast conserving surgery (BCS); ii) the odds of not receiving radiation therapy after BCS versus receiving follow-up radiation therapy. Covariates included race/ethnicity, age at diagnosis, tumour grade, tumour sequence, year of diagnosis, census tract-level poverty rate and urban/rural residence. The spatial scan statistic method was used to identify geographic areas with significantly higher likelihood of experiencing mastectomy. The study found that geographic accessibility to radiation therapy facilities was negatively associated with the likelihood of receiving mastectomy after adjustment for other covariates, but not associated with radiation therapy use among patients receiving BCS. Compared with patients travelling less than 30 minutes to a radiation therapy facility, patients travelling more than 90 minutes were about 1.5 times more likely to receive mastectomy (odds ratio, 1.51 ; $95 \%$ confidence interval, 1.08-2.11) and patients travelling more than 120 minutes were 1.7 times more likely to receive mastectomy (odds ratio, 1.70; 95\% confidence interval, 1.19-2.42). The study also identified a statistically significant cluster of patients receiving mastectomy who were located in south-eastern South Dakota, after adjustment for other factors. Because geographic proximity to treatment facilities plays an important role on the treatment for early-stage breast cancer patients, this study has important implications for developing targeted intervention to reduce disparities in breast cancer treatment in South Dakota.

\section{Introduction}

Breast cancer is the most common cancer and the second leading cause of death among women in the United States (U.S. Cancer Statistics Working Group, 2016). According to the American Cancer Society (ACS) there were 252,710 new cases of invasive breast cancer in 2017 (ACS, 2017). Early-stage invasive breast cancer refers to breast cancer that has not spread to distant parts of the body. There are two types of common treatments for patients with this diagnosis: i) mastectomy; ii) breast conserving surgery (BCS) followed by radiation therapy (ACS, 2016). These two treatments have about the same survival rates among patients (Siegel et al., 2013; Kurian et al., 2014). About $60 \%$ of early-stage patients receive $\mathrm{BCS}$ and $36 \%$ patients receive mastectomy in the United 
States (Siegel et al., 2013). Mastectomy is a surgical procedure that removes the entire breast resulting in a low chance of recurrence among early-stage breast cancer patients. Comparatively, BCS is a surgical procedure that removes the tumour and a small rim of normal tissue around it. However, this approach requires five to seven weeks of radiation therapy following the surgery (Fisher, 2002). Studies have shown that BCS followed by radiation therapy has a $50 \%$ lower risk of breast cancer recurrence and 20\% lower risk of death compared with BCS alone without radiation therapy (Darby et al., 2011). The local recurrence rate is related to tumour characteristics, but the overall 10-year local recurrence rate is similar $(10 \%$ $20 \%$ ) among early-stage breast cancer patients after a mastectomy or BCS with radiation therapy (Freedman and Fowble, 2000). BCS followed by radiation therapy is the recommended course of action for the majority of early-stage breast cancer patients (National Institutes of Health (NIH), 1991). Multiple factors might be associated with breast cancer treatments among early-stage patients, including tumour/cancer characteristics, doctor's preference, patient factors, and hospital/treatment facility factors. Examples of tumour/cancer characteristics include tumour size, tumour location, and margins (the rim of normal tissue surrounding the tumour). BCS is usually conducted if there is only one site of cancer in the breast and the tumour size is under $4 \mathrm{~cm}$. BCS also requires clear margins with no cancer cells in the tissue surrounding the tumour. In contrast, mastectomy should be conducted for patients with a large tumour, two or more tumours in different areas of the breast, or no clear margins (NIH, 1990). A doctor's preference toward certain treatment might be a factor as well. Some doctors might recommend mastectomy to patients when they believe mastectomy has a lower chance of recurrence for patients (Smith et al., 2009).

Individual patient factors may also affect cancer treatment choices. Examples include age, education level, awareness about cancer treatment choices or patient education related to cancer treatment, socioeconomic factors, past radiation therapy, genetic factors, fearfulness of recurrence and willingness to commit to the daily schedule of radiation therapy (Smith et al., 2009; Habermann et al., 2010; Boscoe et al., 2011; Rippy et al., 2014;). The percentage of mastectomy was found to be higher among women $>70$ years of age than that among women $<70$ years of age (Schroen et al., 2005). Another study found that women with lower education levels were less likely to use BCS compared with women with higher education levels (e.g., with college degree) (Smith et al., 2009). Because radiation therapy is usually costly, patients with a low socioeconomic status tend to choose mastectomy (Baldwin et al., 2012). Patients who had previous breast conservations, felt safer about mastectomy or wished to avoid further surgery or future recurrence were also more likely to receive mastectomy (Kirby et al., 2008).

Characteristics of hospitals and other treatment facilities can also influence treatment decisions. Examples of relevant geographic factors include the location and accessibility of treatment facilities relative to the patient's home address. Several previous studies reveal that patients residing in areas without access to radiation therapy centres or needing to travel far were more likely to receive a mastectomy because radiation therapy would require daily trips to a treatment facility (Celaya et al., 2006; Boscoe et al., 2011).

This study aimed to further investigate how geographic access (defined by travel time) to radiation therapy facilities might influence the type of breast cancer treatment received. Few studies of breast cancer treatment have focused on the Northern Plains of the United States (e.g. South Dakota, North Dakota and Wyoming), an area with a high mastectomy rate (Smith et al., 2009; Habermann et al., 2010;). The state of South Dakota is one of the most rural states in the Unites States and about half of its population reside in rural areas (Census, 2010). The age-adjusted incidence rate for breast cancer in South Dakota is 125.9 per 100,000 population and the mortality rate is 20.7 per 100,000 population (ACS, 2016). South Dakota has a higher incidence rate than the United State as a whole (123.1) and other states in the Northern Plains (122.2 in North Dakota, 122.7 in Nebraska and 111.2 in Wyoming). It has been reported that people residing in some of the remotest rural areas of South Dakota, including American Indian reservations, experienced difficulty accessing healthcare facilities (Kanekar and Petereit, 2009). However, it remains unknown whether geographic accessibility to radiation therapy facilities is associated with the receipt of different breast cancer treatments among early-stage patients.

The objective of this study was to examine the association between geographic access to radiation therapy facilities and the receipt of breast cancer treatment among early-stage breast cancer patients. The study adopted a multilevel framework using state-wide population-based data for South Dakota between 2001 and 2012.

\section{Materials and Methods}

\section{Data sources}

Data for this cross-sectional analysis included breast cancer cases diagnosed between 2001 and 2012 obtained from the South Dakota Cancer Registry (SDCR). A total of 4,209 early (localized) stage cases (coded as 1) were extracted from the breast cancer database. Cases who received BCS (codes 20-24) or mastectomy (codes 30-80) were chosen for this study $(\mathrm{n}=4031)$. A total of 178 cases with missing treatment information were removed from the analysis. Covariates included race/ethnicity, age at diagnosis, tumour grade, tumour sequence, year of diagnosis, poverty rate, and urban/rural residence. The poverty rate was extracted from US Census data in 2000, and was measured as the percent of individuals below the federal poverty line in the census tract of residence. The urban/rural residence was defined according to the rural-urban commuting areas (RUCAs) codes (Rural Health Research Center University of Washington, 2010) which placed cases into four categories based on the census tract of residence: urban, large rural city/town, small rural town and isolated small rural town.

The geographic accessibility factor was measured using the shortest travel time from each patient's geocoded residential address to the nearest Radiation Therapy facility using the Network Analyst extension in ArcGIS 10.3.1 (ESRI, Redlands, CA, USA). There were six Radiation Therapy facilities represented in the South Dakota Comprehensive Cancer Control Program (SD CCCP) (SD CCCP 2016). To determine the availably of each facility during 2001 and 2012, we contacted each facility through phone calls and all of them confirmed that they provided radiation therapy services during our study period.

\section{Statistical analysis}

This study used Chi-square tests to examine associations between the type of breast cancer treatment and race/ethnicity, age, tumour grade, tumour sequence, year of diagnosis, poverty, travel time to the closest radiation therapy facility and urban/rural residence. Two-level logistic regression models, which belong to the class of generalized linear mixed models, were used to estimate the following outcomes among early stage cases: i) the odds of mastectomy versus BCS; ii) the 
odds of not receiving radiation therapy after BCS versus receiving following radiation therapy. The generalized linear mixed model allows including both fixed and random effects on the response variable. It also allows the quantification of outcome variation at different levels (e.g., individual and area-level). These characterizes made the generalized linear mixed model suitable for the present study. In the model, patients (level one) were nested within area-level census tracts (level two). Patient-level or individual-level characteristics included race/ethnicity, age at diagnosis, tumour grade, tumour sequence, year of diagnosis and geographic access. Area-level were poverty rate and urban/rural residence at the census tract-level. In order to measure geographic variations of the receipt of treatments in this study, two models were constructed for each multilevel logistic regression model. The first model was an empty model that only had an intercept to estimate the census tract-level random effect of treatment. The second model included all of the covariates, race/ethnicity, age, tumour grade, tumour sequence, year of diagnosis, poverty, travel time to the closest radiation therapy facility and urban/rural residence, along with a census tractlevel random effect. For all analyses, $\mathrm{P}$ values $\leq 0.05$ were considered statistically significant. The two-level models were fit using the GLIMMIX procedure in SAS 9.4 (SAS Institute, Cary, NC, USA).

\section{Geographic variations}

In order to quantify the geographic variations of the receipt of breast cancer treatment, whether the receipt of treatment varies significantly across census tracts, the median odds ratio (MOR) was estimated in this study (Larsen and Merlo, 2005). A larger MOR indicates a larger geographic variation and there is no geographic variation if the MOR is 1 . More details about MOR can be found else- where (Larsen and Merlo, 2005; Lin and Wimberly, 2017).

The spatial scan statistic method was used to identify geographic areas with significantly higher likelihood of mastectomy than BCS. We applied the Bernoulli model, which uses cohorts of cases and controls to determine if there is any significant clustering of the case (patients with mastectomy) location distribution as compared to the controls (patients with BCS) location distribution. The analysis was conducted in the SaTScan (v.9.4.1) software (Kulldorff, 2009). Several covariates found significant in the regression were adjusted for, including age, tumour grade, tumour sequence, geographic proximity to the radiation therapy facility, and poverty level of census tracts, using multiple data sets in SaTScan. In the covariates adjustment, cases and controls were divided into separate datasets based on each covariate. For each dataset log likelihood ratios were calculated for each window location and size first and then summed up. The location and window size at which the log likelihood ratio was maximized was considered the most likely cluster. Statistical testing was carried out using 9999 Monte Carlo simulations to evaluate the significance of candidate clusters (P-value). The relative risk of a cluster was measured by a ratio of the number of observed cases to the number of expected cases within the cluster divided by that ratio outside of the cluster. The expected number of cases in an area was proportional to the sum of cases and controls in that area.

\section{Results}

Figure 1 shows the geographic distribution of travel time to the closest radiation therapy facility in South Dakota. Patients residing

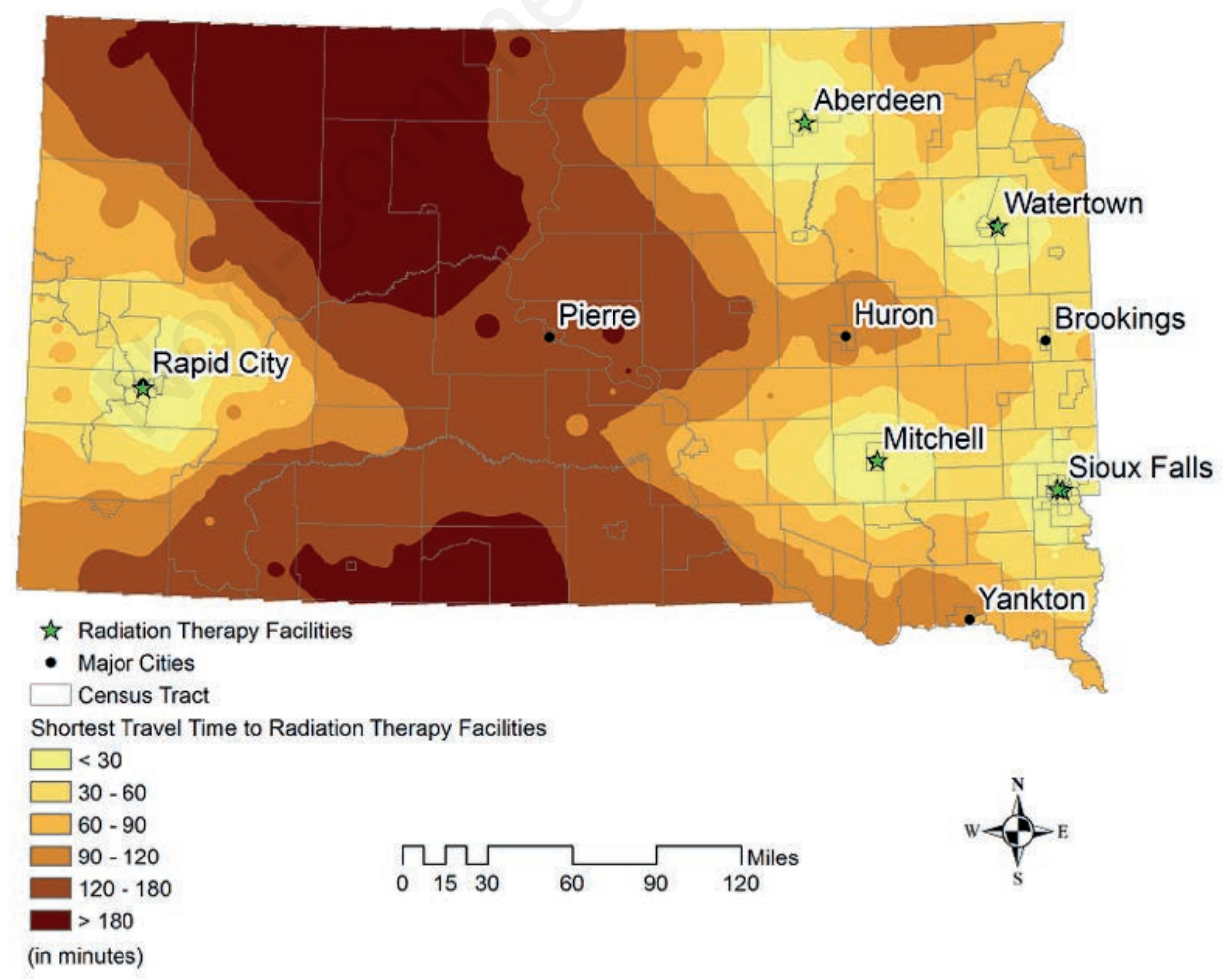

Figure 1. Geographic distribution of travel time to the closest radiation therapy facility.

Map produced using the interpolated travel time from census tracts to the closest radiation therapy facility rather than individual patients' travel times. 
in areas of five major cities where radiation therapy facilities were available, including Sioux Falls, Rapid City, Aberdeen, Watertown and Mitchell, travelled shorter distances to the radiation therapy facilities than patients in the rest of the state. Patients residing in central areas of the state as well as the north-western areas had to travel at least two hours to access a radiation therapy facility.

Table 1 shows the mastectomy and BCS distribution among early stage breast cancer cases by race/ethnicity, age, tumour grade, tumour sequence, year of diagnosis, poverty, travel time to the radiation therapy facility and urban/rural residence. Chi-square tests showed significant differences in all of these individual and area-level characteristics for patients who received a mastectomy versus BCS. For example, a total of $61.6 \%$ of Americans Indians received mastectomy, whereas $47.2 \%$ of the non-Hispanic whites did so. Census tracts with higher poverty rates also had a higher percentage of patients receiving mastectomy compared with tracts with lower poverty rates. The percentage of mastectomy was higher for patients with a longer travel time to a radiation therapy facility than for patients with a shorter travel time. For example, 58.7\% of patients residing in areas with at least two-hour travel time to a radiation therapy facility received mastectomy, whereas $45.2 \%$ of patients residing in areas within a half-hour driving distance to an

Table 1. Characteristics of early stage breast cancer patients receiving mastectomy and breast conserving surgery (BCS) treatments in South Dakota, 2001-2012.

\begin{tabular}{|c|c|c|c|c|c|c|}
\hline \multirow[t]{3}{*}{ Characteristics } & & \multicolumn{4}{|c|}{$\begin{array}{l}\text { Early stage breast cancer } \\
\qquad(\mathrm{n}=4031)\end{array}$} & \multirow{3}{*}{ P value } \\
\hline & & \multicolumn{2}{|c|}{$\begin{array}{l}\text { Mastectomy } \\
(\mathrm{n}=1951)\end{array}$} & \multicolumn{2}{|c|}{$\begin{array}{c}\text { BCS } \\
(n=2080)\end{array}$} & \\
\hline & & Cases & $\%$ & Cases & $\%$ & \\
\hline \multirow[t]{3}{*}{ Race/ethnicity } & Non-Hispanic Whites & 1,725 & 47.2 & 1,928 & 52.8 & \multirow[t]{3}{*}{$<0.001$} \\
\hline & American Indians & 101 & 61.6 & 63 & 38.4 & \\
\hline & Others & 125 & 58.4 & 89 & 41.6 & \\
\hline \multirow[t]{3}{*}{ Age } & $<50$ & 294 & 45.4 & 354 & 54.6 & \multirow[t]{3}{*}{$<0.001$} \\
\hline & $50-64$ & 752 & 55.9 & 594 & 44.1 & \\
\hline & $>64$ & 1,034 & 50.8 & 1,003 & 49.2 & \\
\hline \multirow{6}{*}{ Tumour grade } & & & & & & \multirow{6}{*}{$<0.001$} \\
\hline & Well differentiated & 517 & 44.7 & 640 & 55.3 & \\
\hline & Moderately differentiated & 783 & 45.2 & 950 & 54.8 & \\
\hline & Poorly differentiated & 490 & 56.5 & 377 & 43.5 & \\
\hline & Undifferentiated & 10 & 62.5 & 6 & 37.5 & \\
\hline & Unknown & 151 & 58.5 & 107 & 41.5 & \\
\hline \multirow[t]{3}{*}{ Tumor sequence } & First or only tumor & & & & & \\
\hline & 1,634 & 46.8 & 1,858 & 53.2 & $<0.001$ & \\
\hline & 2nd or subsequent tumor & 317 & 58.8 & 222 & 41.2 & \\
\hline \multirow[t]{4}{*}{ Poverty rate } & $<5 \%$ & 454 & 43.3 & 594 & 56.7 & \multirow[t]{4}{*}{$<0.001$} \\
\hline & $5 \%-10 \%$ & 454 & 47.9 & 493 & 52.1 & \\
\hline & $10 \%-15 \%$ & 462 & 48.5 & 490 & 51.5 & \\
\hline & $>15 \%$ & 581 & 53.6 & 503 & 46.4 & \\
\hline \multicolumn{6}{|c|}{ Travel time to radiation therapy facilities (in minutes) } & \multirow{6}{*}{$<0.001$} \\
\hline & $<30$ & 874 & 45.2 & 1,061 & 54.8 & \\
\hline & $30-60$ & 314 & 46.2 & 366 & 53.8 & \\
\hline & $60-90$ & 277 & 51.5 & 261 & 48.5 & \\
\hline & $90-120$ & 237 & 52.2 & 217 & 47.8 & \\
\hline & $>120$ & 249 & 58.7 & 175 & 41.3 & \\
\hline \multirow[t]{12}{*}{ Year of diagnosis } & 2001 & 204 & 62.8 & 121 & 37.2 & \multirow[t]{12}{*}{$<0.001$} \\
\hline & 2002 & 174 & 54.0 & 148 & 46.0 & \\
\hline & 2003 & 166 & 51.6 & 156 & 48.4 & \\
\hline & 2004 & 128 & 46.9 & 145 & 53.1 & \\
\hline & 2005 & 180 & 51.1 & 172 & 48.9 & \\
\hline & 2006 & 136 & 44.4 & 170 & 55.6 & \\
\hline & 2007 & 174 & 50.1 & 173 & 49.9 & \\
\hline & 2008 & 157 & 45.6 & 187 & 54.4 & \\
\hline & 2009 & 185 & 52.9 & 165 & 47.1 & \\
\hline & 2010 & 134 & 45.3 & 162 & 54.7 & \\
\hline & 2011 & 160 & 40.3 & 237 & 59.7 & \\
\hline & 2012 & 153 & 38.5 & 244 & 61.5 & \\
\hline \multirow{4}{*}{ Urban/rural residence } & Urban & 789 & 46.5 & 908 & 53.5 & \multirow{4}{*}{$<0.001$} \\
\hline & Large rural town & 443 & 44.7 & 548 & 55.3 & \\
\hline & Small rural town & 126 & 55.0 & 103 & 45.0 & \\
\hline & Isolated small rural town & 593 & 53.2 & 521 & 46.8 & \\
\hline
\end{tabular}

The P value was determined by chi-square tests. 
available radiation therapy facility did so.

Table 2 shows multilevel logistic regression results for the odds of receiving mastectomy among early-stage breast cancer patients $(n=4,031)$. The model was adjusted for all of the covariates in the table. Travel time to the closest radiation therapy facility was associated with the odds of receiving mastectomy. Compared to patients with 30-minute or less driving time to a radiation therapy facility, patients residing in areas with travel time between 90 and 120 minutes were $51 \%$ more likely to receive mastectomy (Odds Ratio (OR): $1.51 ; 95 \%$ Confidence Interval (CI): (1.08 - 2.11)). The odds of mastectomy for patients residing in areas with driving time more than two hours was even higher (OR: 1.70; 95\% CI: (1.1-2.42)). Of the other covariates, age, tumour grade, tumour sequence and poverty rate were significantly associated with the odds of mastectomy. Patients diagnosed at an older age $(\geq 50)$ were more likely to experience mastectomy compared with patients diagnosed when younger than 50 years old. Compared with patients living in census tracts with less than $5 \%$ of population under the federal poverty line, patients living in census tracts with greater than $15 \%$ of population under the federal poverty line were more likely to receive

Table 2. Two-level logistic regression model results of the odds of receiving mastectomy relative to breast conserving surgery among early-stage breast cancer patients $(n=4,031)$.

\begin{tabular}{|c|c|c|}
\hline \multicolumn{2}{|l|}{$\begin{array}{l}\text { Characteristics } \\
\text { Fixed Effects }\end{array}$} & Model 1 \\
\hline Fixed Effects & & \\
\hline $\begin{array}{l}\text { Travel time to } \\
\text { radiation therapy } \\
\text { facilities (in minutes) }\end{array}$ & $\begin{array}{l}<30 \\
30-60 \\
60-90 \\
90-120 \\
>120\end{array}$ & $\begin{array}{c}1 \text { (referent) } \\
1.06(0.80-1.41) \\
1.30(0.95-1.77) \\
1.51(1.08-2.11)^{*} \\
1.70(1.19-2.42)^{*}\end{array}$ \\
\hline Race/ethnicity & $\begin{array}{l}\text { Non-Hispanic Whites } \\
\text { American Indians }\end{array}$ & $\begin{array}{c}1 \text { (referent) } \\
1.37(0.96-1.96)\end{array}$ \\
\hline Age & $\begin{array}{l}<50 \\
50-64 \\
>64\end{array}$ & $\begin{array}{c}1 \text { (referent) } \\
1.47(1.22-1.82)^{* *} \\
1.22(1.01-1.47)^{*}\end{array}$ \\
\hline Tumor grade & $\begin{array}{l}\text { Well differentiated } \\
\text { Moderately } \\
\text { differentiated } \\
\text { Poorly differentiated } \\
\text { Undifferentiated } \\
\text { Unknown }\end{array}$ & $\begin{array}{c}1 \text { (referent) } \\
1.04(0.89-1.22) \\
\\
1.61(1.34-1.94)^{* *} \\
1.59(0.55-4.61) \\
1.75(1.31-2.33)^{* *}\end{array}$ \\
\hline Tumor sequence & $\begin{array}{l}2^{\text {nd }} \text { or subsequent } \\
\text { tumor } \\
\text { First or only tumor } \\
<5 \%\end{array}$ & $\begin{array}{c}1 \text { (referent) } \\
0.59(0.49-0.72)^{* *} \\
1 \text { (Referent) }\end{array}$ \\
\hline Poverty & $\begin{array}{l}5 \%-10 \% \\
10 \%-15 \% \\
>15 \%\end{array}$ & $\begin{array}{c}1.06(0.86-1.31) \\
1.10(0.88-1.37) \\
1.28(1.02-1.61)^{*}\end{array}$ \\
\hline Urban/rural residence & $\begin{array}{l}\text { Urban } \\
\text { Large rural town } \\
\text { Small rural town } \\
\text { Isolated small rural town }\end{array}$ & $\begin{array}{c}1 \text { (referent) } \\
0.80(0.61-1.01) \\
1.12(0.73-1.73) \\
0.87(0.64-1.18)\end{array}$ \\
\hline
\end{tabular}

${ }^{*} \mathrm{P}<0.001,{ }^{*} \mathrm{P}<0.05$. Odds ratio was adjusted for all of the factors in the table along with year of diagnosis. The census-tract level variance is 0.20 and MOR is 1.53 in the empty model. mastectomy (OR: 1.28 95\% CI: (1.02-1.61)). Race/ethnicity and urban/rural residence were not significant.

Table 3 shows multilevel logistic regression results for the odds of not receiving radiation therapy after BCS among earlystage breast cancer patients who had BCS $(n=2,080)$. The model was adjusted for all of the covariates in the table. Among 2,080 patients who had BCS, 459 did not receive follow-up radiation therapy. Travel time to the closest radiation therapy facility was not a significant factor influencing radiation therapy use. Among other covariates, an older age $(>64)$ was significantly associated with the odds of not receiving radiation therapy after BCS. Sequence and tumour grade also had significant effects on radiation therapy use after BCS. Of the other covariates, race/ethnicity, census-tract poverty level or urban/rural residence were not significant.

There were significant geographic variations of breast cancer treatment. For mastectomy, the census-tract level variance was 0.20 in the empty model (Table 2). After adjustment for all of the covariates, the census-tract level variance was still significant although it decreased to 0.17 , and there was only a slight decrease in MOR. For BCS without radiation therapy, the census-tract level

Table 3. Two-level logistic regression model results of the odds of not receiving radiation therapy after breast conserving surgery among early-stage breast cancer patients $(n=2,080)$.

\begin{tabular}{llc} 
Characteristics & & Model 1 \\
\hline Fixed Effects & & \\
Travel time to & $<30$ & \\
RT (in minutes) & $30-60$ & $1.05(0.65-1.69)$ \\
1 (referent) & $60-90$ & $1.01(0.59-1.73)$ \\
& $90-120$ & $0.98(0.55-1.74)$ \\
& $>120$ & $1.32(0.70-2.50)$ \\
Race/ethnicity & Non-Hispanic Whites & 1 (referent) \\
& American Indians & $1.37(0.96-1.96)$ \\
\hline Age & $<50$ & 1 (referent) \\
& $50-64$ & $0.86(0.58-1.28)$ \\
& $>64$ & $2.36(1.63-3.40)^{* *}$ \\
Tumour grade & Well differentiated & 1 (referent) \\
& Moderately differentiated & $0.99(0.76-1.31)$ \\
& Poorly differentiated & $1.42(1.01-1.99)^{*}$ \\
& Undifferentiated & $7.94(1.47-42.94)^{*}$ \\
& Unknown & $1.22(0.70-2.13)$ \\
\hline Tumour sequence & $2 n d$ or subsequent tumour & 1 (referent) \\
& First or only tumour & \\
& $0.49(0.35-0.69)^{* *}$ & 1 (referent) \\
& $<5 \%$ & $0.99(0.70-1.42)$ \\
Poverty & $5 \%-10 \%$ & $1.38(0.95-1.99)$ \\
& $10 \%-15 \%$ & $1.22(0.82-1.83)$ \\
\hline Urban/rural residence & $>15 \%$ Urban & 1 (referent) \\
& Large rural town & $0.72(0.47-1.09)$ \\
& Small rural town & $1.22(0.58-2.59)$ \\
& Isolated small rural town & $0.73(0.44-1.24)$ \\
& & \\
& & \\
& & \\
& & \\
& & \\
& &
\end{tabular}

Census-tract level variance 0.22

Median Odds Ratio (MOR) 1.56

${ }^{* *} \mathrm{P}<0.001,{ }^{*} \mathrm{P}<0.05$. Odds ratio was adjusted for all of the factors in the table along with year of diagnosis. The census-tract level variance is 0.25 and MOR is 1.61 in the empty model. 
variance was 0.25 in the empty model (Table 3 ). After adjustment for all of the covariates, the census-tract level variance was still significant and there was only a slight decrease in MOR from 1.61 to 1.56 . The results suggest that unexplained geographic variations of breast cancer treatment exist.

Figure 2 presents geographic clusters of early-stage breast cancer patients receiving mastectomy. Figure 2A shows two geographic clusters before adjustment for covariates. The first cluster, located in the south-eastern part of the state (cluster 1 in Figure 2A), had a relative risk of 1.42. The second cluster (cluster 2 in Figure 2A), observed in south-western South Dakota, covering Pine Ridge and Rosebud Indian reservations, had a relative risk of 1.41. After adjustment for covariates included in the multi-level regression model, cluster 2 disappeared and cluster 1 shrank substantial- ly, although the relative risk remained high (Figure 2B). These results suggest that the cluster in Figure $2 \mathrm{~B}$, which is cantered on the Yankton Indian Reservation, may be explained by other factors not included in the study.

\section{Discussion}

A number of studies have documented the significant effect of travel distance to the treatment centre on receipt of mastectomy (Kerner et al., 2002; Schroen et al., 2005; Celaya et al., 2006; Voti et al., 2006; Boscoe et al., 2011; Goyal et al., 2014; Lam et al., 2015). Affordability and accessibility are the two factors that impact healthcare access (Voti et al., 2006). Affordability is gener-

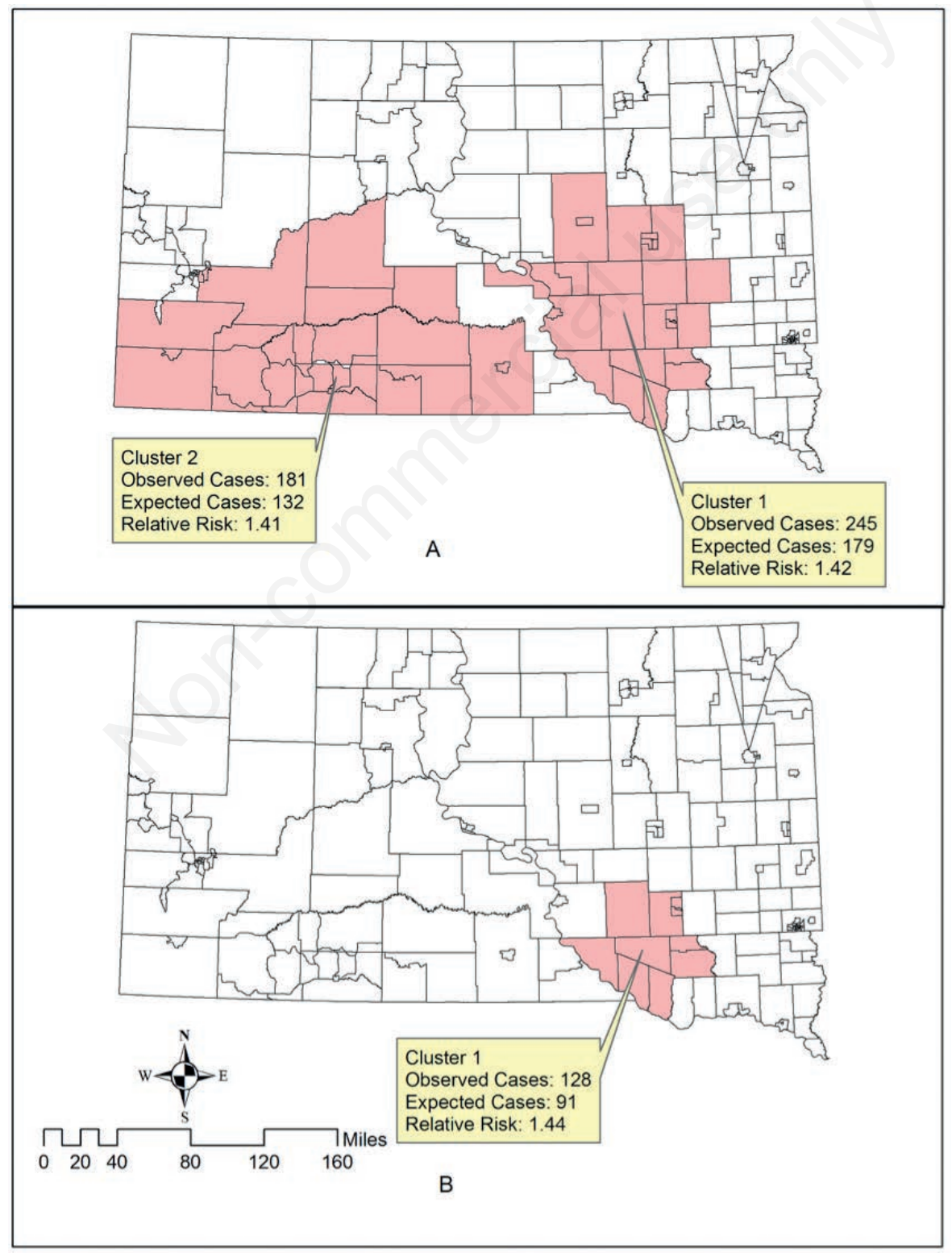

Figure 2. Geographic clusters of early-stage breast cancer patients receiving mastectomy. A) Unadjusted clusters; B) Cluster adjusted for covariates. 
ally associated with socioeconomic factors (e.g., the significance of poverty found in this study) as well as health insurance, while accessibility is associated with geographic factors. We used the shortest travel time to the closest radiation therapy facility as a proxy of the geographic accessibility and our results corroborated previous findings in the literature that longer travel time is significantly associated with the likelihood of receiving mastectomy (Celaya et al., 2006; Boscoe et al., 2011). However, none of these studies examined travel distances as long as those in South Dakota. Because BCS requires several subsequent visits to radiation therapy facilities, whereas mastectomy does not require any subsequent visits to radiation therapy facilities, geographic accessibility might be a potential factor that influences patients'or doctors' decisions in this respect.

This study did not find significant associations between travel time to radiation therapy facilities and the receipt of radiation therapy after BCS. Contradictory findings have been documented in the literature regarding the effect of geographic accessibility on the receipt of radiation therapy following BCS. Some studies found that the receipt of radiation therapy after BCS was significantly related to the distance to the radiation therapy facilities (Athas et al., 2000; Kerner et al., 2002; Celaya et al., 2006; Wheeler et al., 2014; Hsieh et al., 2015; Liu et al., 2015). Similar to the present study, some failed to associate travel time to radiation therapy facilities with the receipt of radiation therapy following BCS (Sauerzapf et al., 2008). One possible explanation is that because radiation therapy is usually required after BCS for patients who have decided to receive $\mathrm{BCS}$, distance or travel time to radiation therapy no longer influences the receipt of radiation therapy. Further examination of data revealed that about $70 \%$ of patients who chose BCS lived close (within 60 miles) to radiation therapy facilities. Only 437 out of 2,048 patients $(21.3 \%)$ who received BCS did not receive follow-up radiation therapy. These patients were older $(>64)$ with poorly-differentiated or undifferentiated tumour grades or had a second or subsequent tumour in the present study.

Patients living in areas with more than $15 \%$ of the population below the federal poverty line were more likely to receive mastectomy compared with patients living in areas with less than $5 \%$ below the federal poverty line. This finding was consistent with previous studies (Kerner et al., 2002; Boscoe et al., 2011; Baldwin et al., 2012). It has been documented that socioeconomically disadvantaged patients were more likely to have larger or more advanced tumours for which mastectomy was often recommended (Akinyemiju et al., 2016). Another possible explanation of the association between poverty level and the receipt of treatment is the cost of treatment. Because BCS has significantly higher shortterm costs than mastectomy for early-stage breast cancer (Barlow et al., 2001), patients with socioeconomic disadvantage (e.g., low income, no insurance) might choose mastectomy over BCS.

This study found that older age was a significant predictor of the receipt of mastectomy. Similar findings were reported in previous studies (Voti et al., 2006; Boscoe et al., 2011). It is well documented that older patients are more likely to have more advanced tumours which require more aggressive treatments (Sauerzapf et al., 2008). Comorbidity might also explain the receipt of mastectomy among older patients. Furthermore, older patients might be more likely to have a history of breast cancer, which made them less likely to receive BCS. One study suggested that older patients were less body-conscious. Younger patients worried more about their image and preferred BCS to keep a cosmetically acceptable breast compared with older patients (Rippy et al., 2014).

Among women who received BCS, older women were less likely to receive radiation therapy. Previous studies also found that about one third of older women did not receive radiation therapy following BCS (Ballard-barbash et al., 1996; Kerner, 2002). Studies have found that among older patients, the receipt of radiation therapy decreased as the comorbidity increased (Ballard-barbash et al., 1996). The receipt of radiation therapy is also associated with the tumour sizes and patients with tumour size larger than $5 \mathrm{~cm}$ were less likely to receive radiation therapy. The perception of possible side effects brought by radiation therapy among physicians and older patients might also explain the lower radiation therapy rate among older patients.

Results from this study suggest that unexplained geographic variations of breast cancer treatment still exist. Several studies found that other factors, such as quality and equipment of hospitals, hospital reputation, referral factors and surgeon factors, contribute to the geographic disparities of cancer treatment (Mor et al., 2000; Kirby et al., 2008; Anderson et al., 2015). However, relevant data were not available in this study to allow further examination of the geographic variations. Future studies on the impact of hospitals and surgeons are needed.

Several limitations of this study need to be considered. First, the choice of breast cancer treatment among early-stage patients might be impacted by several other factors, including tumour size, location of the tumour, margins, doctors' biases toward certain treatment, patients' education levels, insurance, family history and psychological factors (e.g., fear of recurrence of cancer). This study did not include the above factors because data were not available. The unexplained geographic cluster of mastectomy might be explained by these above factors not included in the present study. Second, geographic access to radiation therapy facilities was measured by the travel time from patients to the closest radiation therapy facilities rather than the actual radiation therapy facilities, but this information was not available to us. It is possible that the closest radiation therapy facility may not necessarily be the one patients actually used. The present study also assumed that travel time was based on travelling by car under the maximum allowable speed, which failed to consider different travel modes or road conditions (e.g., snow in the winter). Third, all of the treatment facilities included in the present study provided radiation therapy services during our study period (2001-2012). However, possible changes at each facility (e.g., new physicians or equipment) during this period might have impacted the results from this study. We were not able to examine the impact of healthcare quality of each facility on treatment outcomes in the present study due to a lack of data. Another limitation is the use of census poverty data from 2000 and RUCA data from 2010, which might fail to capture changes during the 12 years of the study. Finally, the edge effect or border effect might have influenced our results. We only included radiation therapy facilities within the state boundary of South Dakota. However, border crossing is an issue since patients can travel outside of South Dakota for treatment. We further examined the radiation therapy facilities in neighbouring states, including North Dakota, Wyoming, Nebraska, Iowa and Minnesota. We found that most radiation therapy facilities in neighbouring states are at least three hours travel from the border. Thus, it seems unlikely that patients residing in South Dakota to travel out of state to receive treatment since instate treatment facilities are much closer.

Despite the limitations discussed above, this study has several significant strengths. First, the study examined the impact of geo- 
graphic access to radiation therapy facilities on the receipt of breast cancer treatment. It has been reported that residents in South Dakota experienced great geographic obstacles to access cancer treatment facilities because of the long distances that must be travelled (Kanekar and Petereit, 2009). To our knowledge, this is the first study to examine the statistical association between geographic accessibility and breast cancer treatment based on the state's cancer registry data in South Dakota. Our results corroborate the prevailing assumption that the rural and American Indian populations in South Dakota experience barriers of geographic access to radiation therapy facilities that impacted the choice of treatment among early-stage breast cancer patients. This finding provides useful information for targeted interventions regarding better healthcare resource allocation in South Dakota, such as patientcantered care that involves home counselling on treatment options.

Second, the study also examined small-area (census tract level) geographic disparities of breast cancer treatment. Few studies have been conducted on geographic disparities of cancer treatment and this is the first study on this issue in South Dakota to our knowledge. Moreover, the study examined the joint effect patient-level factors (e.g., race/ethnicity, tumour grade and tumour sequence) as well as contextual-level factors (e.g., urban/rural residence, neighbourhood-level poverty and geographic access) on geographic disparities of breast cancer treatment. Other findings from this study (e.g., geographic clusters of the receipt of mastectomy) provide essential information for the development of geographically targeted intervention on treatment among early-stage breast cancer patients such as free transportation and housing for patients from remote areas.

\section{Conclusions}

This study reveals that early-stage breast cancer patients living farther away from radiation therapy facilities, or residing in areas with high poverty, were more likely to receive mastectomy. In contrast, patient-level factors, not contextual factors, were more important for not receiving radiation therapy after BCS. Our results suggest that there is a need to improve care for early-stage breast cancer patients residing in areas remote to radiation therapy facilities in South Dakota. It might be beneficial for early-stage breast cancer patients to have better access to local treatment services, especially in socioeconomically disadvantaged areas. However, building more treatment centres in rural areas is costly. Other options could include more patient-cantered care. For example, more education and counselling on different treatment options should be helpful. Transportation services (e.g., taxi or car service offered free to patients to and from treatment) or transportation reimbursement for mileage and parking can be provided to patients who live far away from treatment. Free housing for patients and caregiver might also reduce the burden for socioeconomically disadvantaged patients or patients living remotely.

\section{References}

Akinyemiju T, Ogunsina K, Sakhuja S, Ogbhodo V, Braithwaite D, 2016. Life-course socioeconomic status and breast and cervical cancer screening: analysis of the WHO's Study on Global Ageing and Adult Health (SAGE). BMJ Open 6:e012753.
American Cancer Society, 2017. Cancer facts \& figures 2017. Atlanta, GA: American Cancer Society. Available from: https://www.cancer.org/content/dam/cancer-org/research/cancer-facts-and-statistics/annual-cancer-facts-and-figures/ 2017/cancer-facts-and-figures-2017.pdf

American Cancer Society, 2016. Cancer Facts \& Figures 2016: 19. Available from: https://www.cancer.org/content/dam/cancerorg/research/cancer-facts-and-statistics/annual-cancer-factsand-figures/2016/cancer-facts-and-figures-2016.pdf

Anderson RT, Morris CR, Kimmick G, Trentham-Dietz A, Camacho F, Wu X, Lipscomb J, 2015. Patterns of locoregional treatment for nonmetastatic breast cancer by patient and health system factors. Cancer 121:790-9.

Athas WF, Hunt WC, Amir-fazli A, Key CR, 2000. Travel distance to radiation therapy and receipt of radiotherapy following breast-conserving surgery. J Natl Cancer Inst 92:269-71.

Baldwin LM, Patel S, Andrilla CHA, Rosenblatt RA, Doescher MP, 2012. Receipt of recommended radiation therapy among rural and urban cancer patients. Cancer 118:5100-9.

Ballard-barbash R, Potosky AL, Harlan LC, Nayfield SG, Kessler LG, 1996. Factors associated with surgical and cancer in older women, J Natl Cancer Inst 88:716-26.

Barlow WE, Taplin SH, Yoshida CK, Buist DS, Seger D, Brown M, 2001. Cost comparison of mastectomy versus breast-conserving therapy for early-stage breast cancer. J Natl Cancer Inst 93:447-55.

Boscoe FP, Johnson CJ, Henry KA, Goldberg DW, Shahabi K, Elkin EB, Cockburn M, 2011. Geographic proximity to treatment for early stage breast cancer and likelihood of mastectomy. Breast 20:324-8.

Celaya MO, Rees JR, Gibson JJ, Riddle BL, Greenberg ER, 2006. Travel distance and season of diagnosis affect treatment choices for women with early-stage breast cancer in a predominantly rural population (United States). Cancer Causes Control 17:851-6.

Census USB of the 2010 Urban and Rural Population by State, 2010. Available from: http:/www.census.gov/geo/www/ua/ 2010urbanruralclass.html

Darby S, McGale P, Correa C, Taylor C, Arriagada R, Clarke M, Wood W, 2011. Effect of radiotherapy after breast-conserving surgery on 10-year recurrence and 15-year breast cancer death: Meta-analysis of individual patient data for 10801 women in 17 randomised trials. Lancet 378:1707-16.

Fisher B, 2002. Twenty-year follow-up of a randomized trial comparing total for the treatment of invasive breast cancer. N Eng J Med 347:1233-41.

Freedman GM, Fowble BL, 2000. Local recurrence after mastectomy or breast-conserving surgery and radiation. Oncology 14:1561-80.

Goyal S, Chandwani S, Haffty BG, Demissie K, 2014. Effect of travel distance and time to radiotherapy on likelihood of receiving mastectomy. Ann Surg Oncol 22:1095-101.

Habermann EB, Abbott A, Parsons HM, Virnig BA, Al-Refaie WB, Tuttle TM, 2010. Are mastectomy rates really increasing in the United States? J Clin Oncol 28:3437-41.

Hsieh JCF, Cramb SM, McGree JM, Dunn NA, Baade PD, Mengersen KL, 2015. Geographic variation in the intended choice of adjuvant treatments for women diagnosed with screen-detected breast cancer in Queensland. BMC Public Health 15:1204.

Jones AP, Haynes R, Sauerzapf V, Crawford SM, Zhao H, Forman 
D, 2008. Travel time to hospital and treatment for breast, colon, rectum, lung, ovary and prostate cancer. Eur J Cancer 44:992-9.

Kanekar S, Petereit D, 2009. Walking forward: a program designed to lower cancer mortality rates among American Indians in western South Dakota. J South Dakota State Med Assoc 62: 151-9.

Kerner JF, Hadley J, Hwang Y, Eggert L, Johnson LE, Gold K, 2002. Variations in breast carcinoma treatment in older is it black and white? Cancer 95:1401-14.

Kirby RM, Basit A, Manimaran N, 2008. Patient choice significantly affects mastectomy rates in the treatment of breast cancer. Int Sem Surg Oncol 5:20.

Kurian AW, Lichtensztajn DY, Keegan THM, Nelson DO, Clarke CA, Gomez SL, 2014. Use of and mortality after bilateral mastectomy compared with other surgical treatments for breast cancer in California, 1998-2011. Jama 312:902-14.

Lam J, Cook T, Foster S, Poon R, Milross C, Sundaresan P, 2015. Examining determinants of radiotherapy access: do cost and radiotherapy inconvenience affect uptake of breast-conserving treatment for early breast cancer? Clin Oncol 27:465-71.

Larsen K, Merlo J, 2005. Appropriate assessment of neighborhood effects on individual health: integrating random and fixed effects in multilevel logistic regression. Am J Epidemiol 161:81-8.

Lin Y, Wimberly MC, 2017. Geographic variations of colorectal and breast cancer late-stage diagnosis and the effects of neighborhood-level factors. J Rural Health 33:146-57.

Liu E, Santibáñez P, Puterman ML, Weber L, Ma X, Sauré A, Tyldesley S, 2015. A quantitative analysis of the relationship between radiation therapy use and travel time. Int $\mathrm{J}$ Radiat Oncol Biol Phys 93:710-8.

Mor V, Laliberte LL, Petrisek AC, Intrator O, Wachtel T, Maddock
PG, Bland KI, 2000. Impact of breast cancer treatment guidelines on surgeon practice patterns: results of a hospital-based intervention. Surgery 128:847-61.

NIH Consensus Conference. Treatment of early breast cancer. JAMA 1991;265:391-5.

Rippy EE, Ainsworth R, Sathananthan D, Kollias J, Bochner M, Whitfield R, 2014. Influences on decision for mastectomy in patients eligible for breast conserving surgery. Breast 23:2738.

Sauerzapf V, Jones P, Haynes R, Crawford SM, Forman D, 2008. Travel time to radiotherapy and uptake of breast-conserving surgery for early stage cancer in Northern England. Health Place 14:424-33.

Schroen AT, Brenin DR, Kelly MD, Knaus W, Slingluff CL, 2005. Impact of patient distance to radiation therapy on mastectomy use in early-stage breast cancer patients. J Clin Oncol 23:707480.

Siegel R, Desantis C, Virgo K, Stein K, Mariotto A, Smith T, Fedewa S, 2013. Cancer treatment and survivorship statistics. CA Cancer J Clin 62:220-41.

Smith GL, Xu Y, Shih YCT, Giordano SH, Smith BD, Hunt KK, Buchholz T, 2009. Breast-conserving surgery in older patients with invasive breast cancer: current patterns of treatment across the United States. J Am Coll Surg 209:425-33.

Voti L, Richardson LC, Reis IM, Fleming LE, MacKinnon J, Coebergh JWW, 2006. Treatment of local breast carcinoma in Florida: the role of the distance to radiation therapy facilities. Cancer 106:201-7.

Wheeler SB, Kuo T, Durham D, Frizzelle B, Reeder-Hayes K, Meyer A, 2014. Effects of distance to care and rural or urban residence on receipt of radiation therapy among North Carolina Medicare enrollees with breast cancer. North Carolina Med J 75:239-46. 